\title{
Hemodynamic Versus Adrenergic Control of Cat Right Ventricular Hypertrophy
}

\author{
George Cooper IV, Robert L. Kent, Cornelius E. Uboh, Ed W. Thompson, and Thomas A. Marino \\ Cardiology Section of the Department of Medicine, and the Departments of Physiology, Pharmacology, and Anatomy, \\ Temple University School of Medicine, Philadelphia, Pennsylvania 19140
}

\begin{abstract}
The purpose of this study was to determine whether cardiac hypertrophy in response to hemodynamic overloading is a primary result of the increased load or is instead a secondary result of such other factors as concurrent sympathetic activation. To make this distinction, four experiments were done; the major experimental result, cardiac hypertrophy, was assessed in terms of ventricular mass and cardiocyte cross-sectional area. In the first experiment, the cat right ventricle was loaded differentially by pressure overloading the ventricle, while unloading a constituent papillary muscle; this model was used to ask whether any endogenous or exogenous substance caused uniform hypertrophy, or whether locally appropriate load responses caused ventricular hypertrophy with papillary muscle atrophy. The latter result obtained, both when each aspect of differential loading was simultaneous and when a previously hypertrophied papillary muscle was unloaded in a pressure overloaded right ventricle. In the second experiment, epicardial denervation and then pressure overloading was used to assess the role of local neurogenic catecholamines in the genesis of hypertrophy. The degree of hypertrophy caused by these procedures was the same as that caused by pressure overloading alone. In the third and fourth experiments, $\beta$-adrenoceptor or $\alpha$-adrenoceptor blockade was produced before and maintained during pressure overloading. The hypertrophic response did not differ in either case from that caused by pressure overloading without adrenoceptor blockade. These experiments demonstrate the following: first, cardiac hypertrophy is a local response to increased load, so that any factor serving as a mediator of this response must be either locally generated or selectively active only in those cardiocytes in which stress and/or strain are increased; second, catecholamines are not that mediator, in that adrenergic activation is neither necessary for nor importantly modifies the cardiac hypertrophic response to an increased hemodynamic load.
\end{abstract}

\section{Introduction}

Hypertrophy is clearly the basic adaptive response of the heart to hemodynamic overloads. But the important mechanistic question of whether cardiac hypertrophy is a direct cardiocyte

This work was presented in part at the Annual Meetings of the American College of Cardiology, Dallas, TX, 1984, and at the International Society for Heart Research, Oklahoma City, OK, 1984.

Address reprint requests to Dr. Cooper, Cardiology section of the Department of Medicine.

Received for publication 9 October 1984 and in revised form 13 December 1984.

J. Clin. Invest.

(c) The American Society for Clinical Investigation, Inc. $0021-9738 / 85 / 05 / 1403 / 12 \quad \$ 1.00$

Volume 75, May 1985, 1403-1414 response to the increased load or is instead an indirect response to an accompanying increase in potentially trophic factors such as neural or adrenal catecholamines is highly controversial.

The view that the increased load itself may be a sufficient or even the primary factor responsible for cardiac hypertrophy in response to hemodynamic overloads is suggested by three lines of evidence. First, increasing the length of isolated, denervated cardiac tissue has the immediate effect of increasing protein synthesis (1). Second, either during $\beta$-adrenoceptor blockade (2) or after sympathectomy (3), chronic hypoxia, which may result in numerous changes including pulmonary arterial hypertension, causes right ventricular hypertrophy. Third, we have found recently that changing myocardial load, without selective changes either in cardiac innervation or in catecholamine content, results in marked, reversible alterations in cardiac structure and function $(4,5)$. This latter finding, if applicable to the entire potential spectrum of cardiac loading conditions, implies that increased load may be at least a sufficient cause for cardiac hypertrophy.

The studies suggesting that the adrenoceptor activation accompanying a hemodynamic challenge might be the primary factor initiating and maintaining cardiac hypertrophy have been reviewed recently, both in terms of increased cardiac sympathetic nerve activity $(6,7)$ and in terms of elevated levels of circulating adrenal catecholamines $(8,9)$. In the first instance, the reviews $(6,7)$ suggest that increased sympathetic nerve activity is the final common pathway in most or even all situations where an increased hemodynamic load results in cardiac hypertrophy. In the second instance, the reviews $(8,9)$ consider but do not espouse a role for circulating catecholamines in cardiac hypertrophy. Several more recent studies do, however, suggest that circulating adrenal catecholamines may serve the primary hypertrophy mediating role. These include both the observation in vivo that an elevated level of the adrenal catecholamine epinephrine may be a necessary condition for pressure overload hypertrophy (10) and the observation in vitro that the $\alpha_{1}$ activity of exogenous catecholamines stimulates the growth of cultured neonatal cardiocytes $(11,12)$.

The view that another, unknown trophic factor is responsible for initiating cardiac hypertrophy has also been proposed recently (13). In this instance, actively hypertrophying cardiac tissue is thought to synthesize a water soluble, extractable molecule that causes increased RNA and protein synthesis when introduced into the coronary circulation of other hearts, even when these other hearts are mechanically unloaded. Finally, a number of other circulating substances, such as thyroxine, adrenocorticoids, insulin, and growth hormone may be of variable importance as cofactors for cardiac hypertrophy (9), but there is no evidence either that any of these substances initiates cardiac hypertrophy after a hemodynamic overload or that any of these substances causes load-independent cardiac hypertrophy, even when the blood level of such factors is substantially increased during a primary disease of the specific organ of origin. 
The present study tested the hypothesis that increased load itself, rather than such secondary factors as sympathetic activation, may be directly responsible for cardiac hypertrophy in response to hemodynamic overloads. This hypothesis was tested in two ways: first, in order to dissect the specific local effect of load on the initiation and maintenance of hypertrophy from the general effect of neural or circulating agents on the hypertrophy of an entire stressed chamber, cardiocyte size was measured in a small ventricular segment that was unloaded either during or after overloading of the chamber as a whole; second, the possibility that activation of either the $\alpha$-adrenoceptors or the $\beta$-adrenoceptors in a stressed chamber might mediate hypertrophy was tested in terms of cell and chamber size by imposing a ventricular pressure overload either after ventricular denervation or during the chronic blockade of each of these adrenoceptors.

\section{Methods}

\section{Preparation of the experimental models}

Cardiac unloading. The purpose of this procedure was to unload completely a single papillary muscle within an otherwise normally loaded cat right ventricle; the adjacent papillary muscles and the right ventricular free wall served as control tissues. Extensive details of this procedure have been published previously (4). Briefly, adult cats of either sex weighing 1.7-3.8 $\mathrm{kg}$ were fully anesthetized with ketamine hydrochloride $(25 \mathrm{mg} / \mathrm{kg}$ i.m.) and then paralyzed with succinylcholine $(1 \mathrm{mg} / \mathrm{kg}$ i.v.) before intubation and ventilation with $100 \%$ oxygen. Under sterile conditions, a right thoracotomy and then a right ventriculotomy were performed. During brief venous inflow occlusion, the chordae tendinae of a single, thin papillary muscle were transected. After this, the ventriculotomy was closed, venous inflow occlusion was released, the thoracotomy was repaired, and the cats were allowed to recover. These cats, identified as the unloaded group, were studied as two separate subgroups at either 1 or $\mathbf{2}$ wk after this procedure.

Cardiac overloading. Right ventricular hypertrophy was induced by partially occluding the pulmonary artery with a $3.5-\mathrm{mm}$ internal diameter band. Again, full details of this procedure have been published before (14). Cats weighing 1.9-4.7 kg were prepared, and a thoracotomy was performed. The band then was placed around the proximal pulmonary artery. These cats, identified as the banded group, were studied as two separate subgroups at either 2 or 8 wk after this procedure.

Combined cardiac unloading and overloading. These surgical procedures were designed to differentially load two regions of the same cat right ventricle: a single papillary muscle was completely unloaded as described above for the unloaded group, and the remaining right ventricular tissue was pressure overloaded as described above for the banded group. To study both the induction of hypertrophy and the maintenance of hypertrophy, two loading sequences were employed. To study cardiac hypertrophy induction, a group was prepared which consisted of cats weighing $1.6-3.3 \mathrm{~kg}$ in which the unloading and overloading procedures were performed simultaneously as a single operation. These cats, identified as the unloaded-banded group, were studied as two separate subgroups at either 1 or $2 \mathrm{wk}$ after this procedure. To study cardiac hypertrophy maintenance, a second group was prepared which consisted of cats weighing $2.2-3.3 \mathrm{~kg}$; these cats were overloaded in a first operation as described above and then, in a second operation 2 wk later, a papillary muscle in the same right ventricle was unloaded, while the pulmonary artery band remained in place. These cats, identified as the banded-unloaded group, were studied at 1-4 wk after the second surgical procedure.

Control. A fourth group of sham-operated control cats weighing 1.6-4.6 kg was prepared by performing a thoracotomy, pericardiotomy, and dissection of the proximal pulmonary artery from the aortic root.
These cats, identified as the control group, were studied $\sim 1$ mo after surgical preparation.

\section{Treatment of the experimental models}

Cardiac denervation. These procedures were used to delineate the contribution of the intrinsic cardiac nerves to the development of cardiac hypertrophy. This was done by denervating the right ventricles of cats weighing $2.2-3.6 \mathrm{~kg}$ before the cardiac overloading procedure described above. After the same anesthesia and surgical approach described before, the pericardium was widely incised. Just before cardiac denervation, a right ventricular free wall biopsy was obtained, and the tissue was frozen immediately in liquid nitrogen for subsequent norepinephrine determination. Right ventricular denervation was then produced by local application of an $85 \%$ aqueous solution of phenol $(15,16)$. This solution was applied to the pulmonary conus and around the atrioventricular groove below the right atrium with a fine-tipped brush. 1 wk later, a second right ventricular biopsy was obtained during a similar operative procedure; immediately thereafter, the right ventricle was pressure overloaded as described for the banded group. $2 \mathrm{wk}$ after this second procedure, a third right ventricular biopsy was obtained as part of the terminal study.

Cardiac $\beta$-adrenoceptor blockade. This first pharmacological intervention was used to assess the potential role of $\beta$-adrenergic catecholamines in the mediation of load-related cardiac hypertrophy. Cats weighing 2.0-3.2 kg were treated with propranolol hydrochloride $(25$ $\mathrm{mg} / \mathrm{kg}$ i.p.) twice daily for $1 \mathrm{wk}$ before, during, and for $2 \mathrm{wk}$ after the cardiac overloading procedure described above. The adequacy of $\beta$ adrenoceptor blockade was assessed by maximally challenging each cat with a $\beta$-adrenoceptor agonist at terminal study 2 wk after the overloading procedure. This was done by first obtaining an initial heart rate by electrocardiography in cats lightly anesthetized with ketamine hydrochloride $(10 \mathrm{mg} / \mathrm{kg}$ i.m.), and then obtaining the heart rate response, as a percentage of the initial heart rate, to a continuous infusion of isoproterenol $(1.5 \mu \mathrm{g} / \mathrm{kg}$ per min i.v.). This dose of isoproterenol was chosen by giving the concurrently studied control cats a graded infusion of increasing doses of this drug until maximum cardiac acceleration was obtained; when this dose was maintained for more than a few minutes, lethal arrhythmias resulted. This maximally effective dose of the drug was then used as described above in the experimental cats with $\beta$-adrenoceptor blockade.

Cardiac $\alpha$-adrenoceptor blockade. This second pharmacological intervention was used to assess the potential role of $\alpha$-adrenergic catecholamines in the mediation of load-related cardiac hypertrophy. Cats weighing 2.7-3.8 kg were treated with terazosin hydrochloride $(0.6 \mathrm{mg} / \mathrm{kg}$ i.p.) twice daily for $1 \mathrm{wk}$ before, during, and for $2 \mathrm{wk}$ after the cardiac overloading procedure described above. The adequacy of the $\alpha$-adrenoceptor blockade was determined by challenging each cat with an $\alpha$-adrenoceptor agonist at terminal study $2 \mathrm{wk}$ after the overloading procedure. This was done by first obtaining through a stiff, fluid-filled catheter in cats lightly anesthetized with ketamine hydrochloride $(10 \mathrm{mg} / \mathrm{kg}$ i.m.) an initial mean arterial pressure and then obtaining the mean arterial pressure response, as a percentage of the initial value, to a continuous infusion of phenylephrine hydrochloride $(0.2 \mathrm{mg} / \mathrm{kg}$ per $\mathrm{min}$ i.v.). This dose of phenylephrine was chosen, in a manner analogous to that just described for isoproterenol, by finding the dose which gave a maximum pressor response in concurrently studied control cats. Here too, arrhythmic toxicity occurred rapidly at this dose in the control cats.

\section{Evaluation of the experimental models}

General characteristics. At the time of terminal study, the cats were anesthetized as described above; right ventricular pressures were obtained by using a stiff, fluid-filled catheter which was inserted through the right external jugular vein and attached to a strain gauge, with the mid-chest position taken as the zero reference point. After these pressure measurements, a left thoracotomy was done and right ventricular tissue was removed for the determination of norepinephrine; after this, in most cats, the ascending aorta was cannulated for 
perfusion-fixation of the heart through the coronary vascular bed. After fixation, described more fully below, the heart was removed, and the right ventricular free wall and the left ventricle, inclusive of the interventricular septum, were weighed separately. Right ventricular hypertrophy was assessed on the organ level from the ratio of the right ventricular free wall weight to body weight. The ratio of left ventricular weight to body weight was used to assess any potential independent contribution of a change in body weight to the measurement of right ventricular mass. To determine whether either tricuspid valvular incompetence or congestive heart failure was present, the right atrial pressure waveform, the right ventricular end-diastolic pressure, and the liver-to-body weight ratio were examined.

Morphological characteristics. The following procedure was used for perfusion-fixation of the heart. After the hemodynamic determinations described above, each cat was injected with sodium heparin $(1,000 \mathrm{U}$ i.v.), the aorta was cannulated, and the heart was perfused for $3 \mathrm{~min}$ at $100 \mathrm{mmHg}$ pressure with oxygenated Locke's solution containing $2 \%$ procaine in order to arrest it in diastole and clear it of blood. The perfusate was then changed to sodium cacodylate buffer containing $1.5 \%$ glutaraldehyde $(\mathrm{pH}=7.4$; osmolarity $=320$ mosmol) this perfusion lasted for $10 \mathrm{~min}$ (17). After the heart was removed, dissected, and weighed as described above, the papillary muscles from the right ventricle were prepared for electron microscopy by procedures which we have described previously $(17,18)$. From the midportion of each papillary muscle, thick sections $(1 \mu \mathrm{m})$ and thin sections $(60-90$ $\mathrm{nm}$ ) were cut perpendicular to the long axis of the papillary muscle and its constituent cardiocytes. The thin sections were stained with uranyl acetate (19) and lead citrate (20) for the qualitative examination of cardiocyte fine structure by transmission electron microscopy.

The thick sections were stained with toluidine blue for light microscopy and used for assessing right ventricular hypertrophy on the cellular level by the planimetric measurement of cardiocyte crosssectional areas at a magnification of 1,600. Only cardiocyte crosssections which included a centrally located nucleus were used for this measurement. Two blocks were prepared for each papillary muscle, fifty cross-sections were measured with a coefficient of variation of $\sim 20 \%$ in each block, and at least four cats from each group were studied.

Tissue norepinephrine. The tissue samples for this determination were obtained either from the surgical models at terminal study or from the denervated model as biopsy specimens at the previously noted intervals. Each tissue sample was frozen immediately in liquid nitrogen and stored thereafter at $-70^{\circ} \mathrm{C}$. In order to obviate interassay variation, norepinephrine was extracted (21) on a single day from all of these cardiac tissue samples. Each tissue extract also was frozen immediately in liquid nitrogen and stored at $-70^{\circ} \mathrm{C}$. All of the extracts were then analyzed on a second single day by liquid chromatography coupled with electrochemical detection (22).

Norepinephrine was extracted from the tissue as follows. Each frozen tissue sample, weighing $0.5-20.0 \mathrm{mg}$, was minced in $0.3 \mathrm{ml}$ of ice-cold $0.1 \mathrm{~N} \mathrm{HClO}_{4}$ containing $0.4 \mathrm{mM}$ sodium metabisulfite and $0.2 \mathrm{mM}$ EDTA; the internal standard was $20.0 \mathrm{ng}$ of dihydroxybenzylamine in the same volume. Each specimen was homogenized in a small glass mortar and pestle, transferred to a $1.5-\mathrm{ml}$ plastic centrifuge tube, sonicated at $0^{\circ}-5^{\circ} \mathrm{C}$ for $5 \mathrm{~min}$, and centrifuged at $15,600 \mathrm{~g}$ for $20 \mathrm{~min}$. To this supernatant in a fresh tube was added $1 \mathrm{ml}$ of icecold $0.5 \mathrm{M}$ Tris buffer at $\mathrm{pH} 8.6$ containing $3 \mathrm{mM} \mathrm{NaHSO} \mathrm{N}_{3}$ and 0.2 mM EDTA. Next, $20 \mathrm{mg}$ of acid-washed alumina was added to the supernatant and agitated by rotation for $15 \mathrm{~min}$ in order to adsorb catecholamines to the alumina (23). After a 2-min centrifugation, the supernatant was carefully pipetted off of the alumina. The alumina then was treated three times with 1-ml portions of a wash solution containing $1.0 \mathrm{mM} \mathrm{NaHSO}$ in distilled, deionized water. After this, $0.3 \mathrm{ml}$ of $0.05 \mathrm{M}$ ice-cold perchloric acid was added to the alumina and agitated by rotation for $20 \mathrm{~min}$ in order to desorb the norepinephrine from each sample into a minimal volume of acid, which was frozen immediately at $-70^{\circ} \mathrm{C}$ as noted above.

The norepinephrine in these acid extracts was quantified by chro- matography as follows. The liquid chromatograph was equipped with a reverse-phase column $25 \mathrm{~cm}$ long and $4.6 \mathrm{~mm}$ in diameter which was packed with $5-\mu \mathrm{m}$ spheres of octyl dodecyl sulfate. Each tissue sample was thawed on ice and injected into the column through a 20$\mu 1$ loop. The mobile phase, containing $60 \mathrm{mM}$ citric acid, $40 \mathrm{mM}$ $\mathrm{Na}_{2} \mathrm{HPO}_{4}$, and $0.1 \mathrm{mM}$ sodium octyl sulfate, was pumped through the column at a rate of $1.3 \mathrm{ml} / \mathrm{min}$. The amperometric detector was set at a potential of $+0.75 \mathrm{~V}$ with respect to a $\mathrm{Ag} / \mathrm{AgCl}$ reference electrode and calibrated at $2 \mathrm{nA} / \mathrm{volt}$, with a full-scale recorder deflection of $1 \mathrm{~V}$. The norepinephrine and dihydroxybenzylamine from each sample were determined from linear regression of the peak heights of concurrently chromatographed standards. The amount of norepinephrine in each sample was corrected for by the recovery of dihydroxybenzylamine in each sample and then divided by the wet weight of the sample to obtain norepinephrine content.

\section{Statistical analysis}

Each value in this study is expressed as a mean \pm SE. When control and experimental papillary muscles were obtained from the same ventricle and analyzed together, comparisons of the two were made with a paired $t$ test. When control and experimental data were from different cats, the values were compared via an unpaired $t$ test. When one experimental variable was evaluated in more than two groups, a one-way analysis of variance (ANOVA) ${ }^{1}$ was done; if a significant difference was found, this was followed by a post-ANOVA NewmanKeuls test. In all cases, a significant difference was said to exist when $P$ was $<0.05$.

\section{Results}

\section{Load regulation of hypertrophy}

There were four groups of cats in which surgical manipulation of cardiac load was the only experimental intervention. These consisted of groups in which cardiac unloading, cardiac overloading, or combinations of these two procedures were done. For the unloaded group, the data in Table I show that the unloading procedure produced neither right ventricular hypertrophy nor right ventricular failure when the appropriate measures from this group are compared with those from the sham-operated control group. Specifically, the right ventricular systolic pressure and the ratio of right ventricular weight to body weight were normal, as were the right ventricular enddiastolic pressure and the liver-to-body weight ratio. For the overloaded, banded group, the data in Table I indicate that pulmonary artery banding produced, in comparison to control, a substantial increase in right ventricular systolic pressure with a concomitant increase in right ventricular mass. Again, there was no evidence of right ventricular failure. For the two groups in which a combined procedure was done, both the unloadedbanded group, in which the two procedures were performed simultaneously, and the banded-unloaded group, in which banding preceded unloading, demonstrate right ventricular hypertrophy without failure. A selective effect of each of these four surgical procedures on the right ventricular myocardium is indicated by the relative invariance of the ratio of left ventricular weight to body weight. In addition, there was no evidence in any group of tricuspid valvular incompetence with resultant volume overload of the right ventricle, either in terms of an abnormal right atrial pressure waveform during systole or in terms of an elevated liver-to-body weight ratio. Thus, three separate myocardial loading conditions were created: first, a segment of right ventricular myocardium was unloaded

1. Abbreviation used in this paper: ANOVA, analysis of variance. 
Table I. General Characteristics of the Experimental Models

\begin{tabular}{|c|c|c|c|c|c|c|c|c|}
\hline & Control & Unloaded & Banded & $\begin{array}{l}\text { Unloaded- } \\
\text { banded }\end{array}$ & $\begin{array}{l}\text { Banded- } \\
\text { unloaded }\end{array}$ & $\begin{array}{l}\text { Denervated } \\
\text { + banded }\end{array}$ & $\begin{array}{l}\text { Beta blocked } \\
+ \text { banded }\end{array}$ & $\begin{array}{l}\text { Alpha blocked } \\
\text { + banded }\end{array}$ \\
\hline Body wt $(k g)$ & $2.78 \pm 0.26$ & $2.48 \pm 0.11$ & $3.40 \pm 0.23$ & $2.47 \pm 0.11$ & $2.90 \pm 0.24$ & $3.03 \pm 0.21$ & $2.67 \pm 0.12$ & $3.21 \pm 0.15$ \\
\hline $\begin{array}{l}\text { RV-to-body wt } \\
\text { ratio }(g / k g)\end{array}$ & $0.77 \pm 0.04$ & $0.80 \pm 0.04$ & $1.20 \pm 0.05^{*}$ & $1.19 \pm 0.07^{*}$ & $1.10 \pm 0.03^{*}$ & $1.21 \pm 0.06^{*}$ & $1.18 \pm 0.05^{*}$ & $1.14 \pm 0.07^{*}$ \\
\hline $\begin{array}{l}\text { LV-to-body wt } \\
\text { ratio }(g / k g)\end{array}$ & $2.88 \pm 0.12$ & $2.46 \pm 0.12$ & $2.96 \pm 0.28$ & $2.93 \pm 0.18$ & $2.25 \pm 0.10$ & $2.60 \pm 0.07$ & $2.57 \pm 0.20$ & $2.28 \pm 0.25$ \\
\hline $\begin{array}{c}\text { RV systolic } \\
\text { pressure }\end{array}$ & & & & & & & & \\
\hline $\begin{array}{l}(\mathrm{mmHg}) \\
\mathrm{RV} \text { end-diastolic } \\
\text { pressure }\end{array}$ & $19.6 \pm 1.2$ & $18.8 \pm 0.7$ & $51.7 \pm 3.3^{*}$ & $39.6 \pm 3.5^{*}$ & $55.8 \pm 8.1^{*}$ & $55.9 \pm 5.3^{*}$ & $47.4 \pm 6.0^{*}$ & $53.3 \pm 4.4^{*}$ \\
\hline$(\mathrm{mmHg})$ & $2.1 \pm 0.8$ & $2.6 \pm 0.7$ & $4.6 \pm 3.3$ & $4.3 \pm 0.8$ & $4.3 \pm 1.4$ & $4.3 \pm 1.4$ & $4.3 \pm 1.0$ & $4.9 \pm 0.9$ \\
\hline $\begin{array}{c}\text { Liver-to-body wt } \\
\text { ratio }(\mathrm{g} / \mathrm{kg})\end{array}$ & $30.9 \pm 2.4$ & $34.5 \pm 1.1$ & $33.8 \pm 1.7$ & $32.9 \pm 1.8$ & $30.7 \pm 0.6$ & $25.8 \pm 2.0$ & $31.7 \pm 1.5$ & $32.5 \pm 2.3$ \\
\hline Number of cats & 16 & 12 & 17 & 19 & 4 & 7 & 8 & 6 \\
\hline
\end{tabular}

Each value is expressed as a mean $\pm \mathrm{SE}$. Each asterisk indicates a significant difference from both the control group and the unloaded group using a post-analysis of variance Newman-Keuls test at a $P<0.05$. No other significant differences were found to exist. wt, weight.

without a major effect on the remaining right ventricle; second, the entire right ventricle was overloaded; third, differential loading, in which a segment of the right ventricle was unloaded while the remainder was overloaded, was produced.

In each of the surgical models, including the sham-operated controls, the pericardium was incised, and the base of the great vessels and/or the right ventricular free wall were dissected. For this reason, some incidental surgical denervation of the right ventricle was found in all cases. The norepinephrine content of right ventricular papillary muscles from normal cats was $4.92 \pm 0.89 \mathrm{nM} / \mathrm{g}$ wet weight. For the surgical models, the norepinephrine content of all right ventricular regions, including both the free wall and either unloaded or control papillary muscles, was reduced to $\sim 25 \%$ of this normal value at 2 wk after surgery. No significant difference among the models was found.

Cardiocyte cross-sectional area was chosen as the cellular correlate of a change in cardiac tissue mass. The data in Fig. $1 A$ demonstrate that when paired papillary muscles from the same right ventricles are compared, the cardiocytes of unloaded muscles in otherwise normally loaded ventricles became progressively smaller both at 1 and at 2 wk after unloading. When, in contrast, the entire right ventricle was overloaded, the data in Fig. $1 B$ show that there was a progressive increase in cardiocyte cross-sectional area for the overloaded myocardium both at 2 and at $8 \mathrm{wk}$ after pulmonary artery banding.

Unloading of a single papillary muscle in a simultaneously pressure-overloaded right ventricle allowed the potential contribution of factors other than load to the initiation of cardiac hypertrophy to be assessed. That is, if factors other than local cardiocyte stress and/or strain play an important role in initiating cardiac hypertrophy, the unloaded segment of an otherwise overloaded ventricle might not be expected to show the same cardiocyte atrophy noted in Fig. $1 \mathrm{~A}$. However, the data in Fig. $1 C$ demonstrate that when this differential loading was produced, atrophy of the cardiocytes from the unloaded muscles and hypertrophy of the cardiocytes from the adjacent overloaded muscles were apparent both at 1 and at 2 wk after this combined procedure.
Unloading of a single papillary muscle in a previously hypertrophied right ventricle allowed the potential contribution of factors other than load to the maintenance of cardiac hypertrophy to be assessed. That is, if factors other than load play an important role in maintaining hypertrophy, the cardiocytes of the previously hypertrophied and then unloaded papillary muscles would be expected to remain hypertrophied rather than atrophying. The data in Fig. $1 D$, however, demonstrate substantial atrophy of previously hypertrophied papillary muscles when they were unloaded in a right ventricle in which both the pressure overload and the resultant hypertrophy were maintained.

In addition to the gross anatomical changes in cardiac mass and the histological changes in cardiocyte size reported above, the fine structure of myocardium exposed to the four types of surgical load manipulation was examined. The ultrastructure of right ventricular papillary muscle cardiocytes from a sham-operated control cat is shown in Fig. $2 \mathrm{~A}$. The increase in cardiocyte size in the banded right ventricles which was quantified in Fig. $1 B$ is illustrated in Fig. $2 B$; however, the appearance and organization of the organelles of these hypertrophied cardiocytes is not changed from control. The ultrastructure of cardiocytes from an unloaded papillary muscle in an otherwise normally loaded cat right ventricle is shown in Fig. $2 C$. The two major differences from control are the smaller size of the unloaded cardiocytes, as would be expected both from the cardiac mass changes in Table I and from the histological changes in Fig. $1 A$, and the loss of cardiocyte organelles, particularly the myofibrils. The fine structure of papillary muscle cardiocytes which were unloaded at the same time that the remaining right ventricle was overloaded is shown in Fig. $2 \mathrm{D}$. As would be expected from Fig. $1 C$, the cardiocytes were quite small. In addition, the ultrastructure of this tissue is not similar either to that of the control myocardium in Fig. $2 \mathrm{~A}$ or to that of the pressure overloaded myocardium in Fig. $2 B$; instead, it appears to be essentially identical to that of the unloaded tissue in Fig. $2 C$, especially with respect to the myofibrils. In the unloaded cardiocytes of Figs. 2, C and $D$, there is a similar and marked myofibril depletion, as 
A

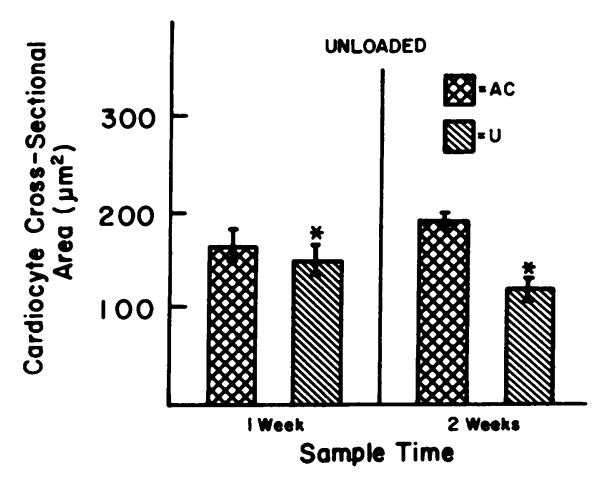

C

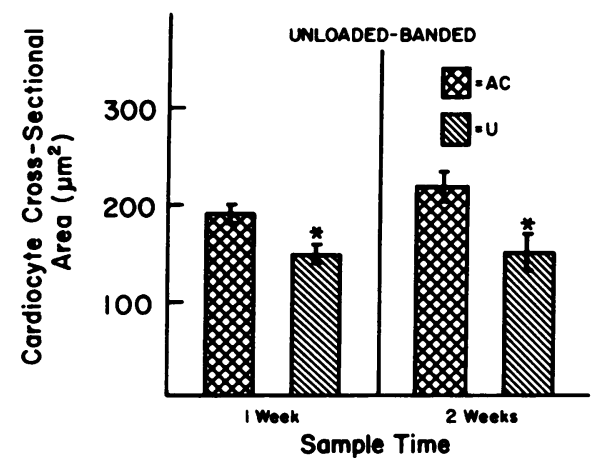

B

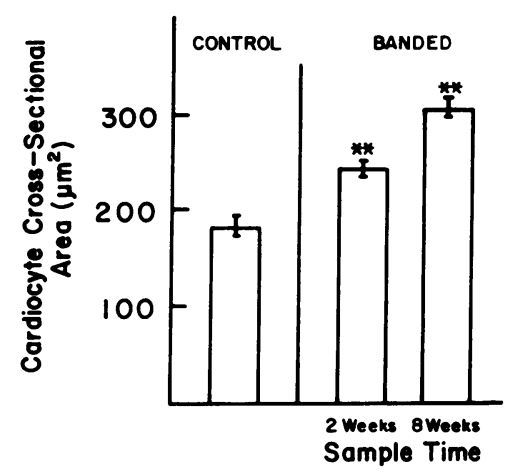

D

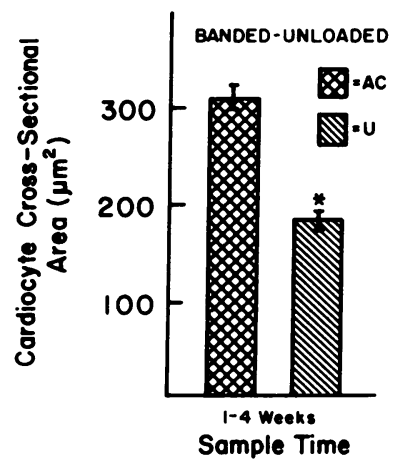

Figure 1. Cardiocyte cross-sectional area as a function of the type of surgical load manipulation. (A) Right ventricular cardiocyte size in unloaded (U) and adjacent control (AC) papillary muscles from the unloaded group at 1 wk after surgery in five cats and at 2 wk after surgery in four cats. (B) Right ventricular cardiocyte size in papillary muscles from 10 sham-operated control cats and in papillary muscles from banded $(B)$ cats at 2 wk after surgery for seven cats and at 8 wk after surgery for 10 cats. $(C)$ Right ventricular cardiocyte size in $U$ and AC papillary muscles from the unloaded-banded group at 1 wk after surgery in seven cats and at 2 wk after surgery in four cats. $(D)$ Right ventricular cardiocyte size in $U$ and AC papillary muscles from four cats in the banded-unloaded group at 1-4 wk after the second procedure; here, the entire right ventricle, with the AC and $U$ papillary muscles, had been caused to hypertrophy by pressure overloading for 2 wk in the first procedure before differential loading was imposed in the second procedure, where the U papillary muscle was unloaded, while the pressure overload on the remaining right ventricle, including the AC papillary muscle, was maintained. There was no significant difference among the four $\mathrm{U}$ groups in $A$ and $C$. Each single asterisk indicates a significant dif-

ference between papillary muscles from the same right ventricles. Each double asterisk indicates a significant difference between papillary muscles from different right ventricles.

well as a loss of the normal orientation of these contractile filaments within the cardiocytes.

\section{Adrenergic regulation of hypertrophy}

There were three groups of cats in which a separate, specific aspect of the myocardial adrenergic input was interrupted before imposing an increased load on the right ventricle. In this way, we examined the potential role of either intrinsic or extrinsic catecholamines in the induction of cardiac hypertrophy in response to an increased load. The three adrenergic interventions consisted of cardiac denervation to remove intrinsic catecholamines and either $\alpha$-adrenergic blockade or $\beta$-adrenergic blockade to obviate the effect of intrinsic and extrinsic catecholamines; a combination of the latter two interventions was prevented by unacceptable mortality. In each case, the effectiveness of the adrenergic intervention was assessed, and it was maintained for 1 wk before and throughout the period of pulmonary artery banding.

The effectiveness of phenol denervation of the cat right ventricle, the first adrenergic intervention, is demonstrated in Fig. $3 A$. The initial control value for the norepinephrine content of the right ventricular free wall immediately before denervation was $6.87 \pm 1.21 \mathrm{nM} / \mathrm{g}$ wet weight, a value in close agreement with that reported previously for cat ventricular myocardium (24). At both 1 and 3 wk after this procedure, the time during which the right ventricle was pressure overloaded, the norepinephrine content of the right ventricular free wall was reduced to $<15 \%$ of control. Thus, there was substantial right ventricular denervation throughout the period when this chamber was pressure overloaded.
When the pulmonary arteries of the denervated cats were banded $1 \mathrm{wk}$ after the denervation procedure, the resultant hypertrophy was similar to that seen with banding alone, as shown both by comparable increases in right ventricular mass in Table I and by comparable increases in cardiocyte crosssectional area in Fig. 3 B. At the ultrastructural level, essentially the same result was observed. In Fig. 4, in which micrographs from both banded and denervated-banded cats are shown along with a micrograph of control tissue, the increase in cardiocyte size is comparable in both of the pressure-overloaded groups. Of further interest is the increase in connective tissue noted in the banded tissue in Fig. $4 \mathrm{~A}$ when compared both with the control tissue in Fig. $4 B$ and to the denervatedbanded tissue in Fig. $4 C$. In addition, the data in Table I indicate that the systolic pressure increment was similar in the two pressure-overloaded groups and that there was no evidence for right ventricular failure in either group.

The effectiveness of $\alpha$-adrenoceptor and $\beta$-adrenoceptor blockade, the interventions designed to block both intrinsic and extrinsic catecholamines, is demonstrated in Fig. $5 \mathrm{~A}$. Since the adrenoceptor blocking agent used in each case was a competitive antagonist, there was a minor response to the very high challenge dosages of each of the respective agonists. However, substantial blockade was achieved in each instance, and it is extraordinarily unlikely that endogenous adrenergic activation could ever approach the levels produced by these toxic pharmacological challenge dosages. The pulmonary arteries of these two groups of cats were banded 1 wk after initiating the appropriate adrenergic blockade, and the degree of resultant hypertrophy which occurred during adrenoceptor 

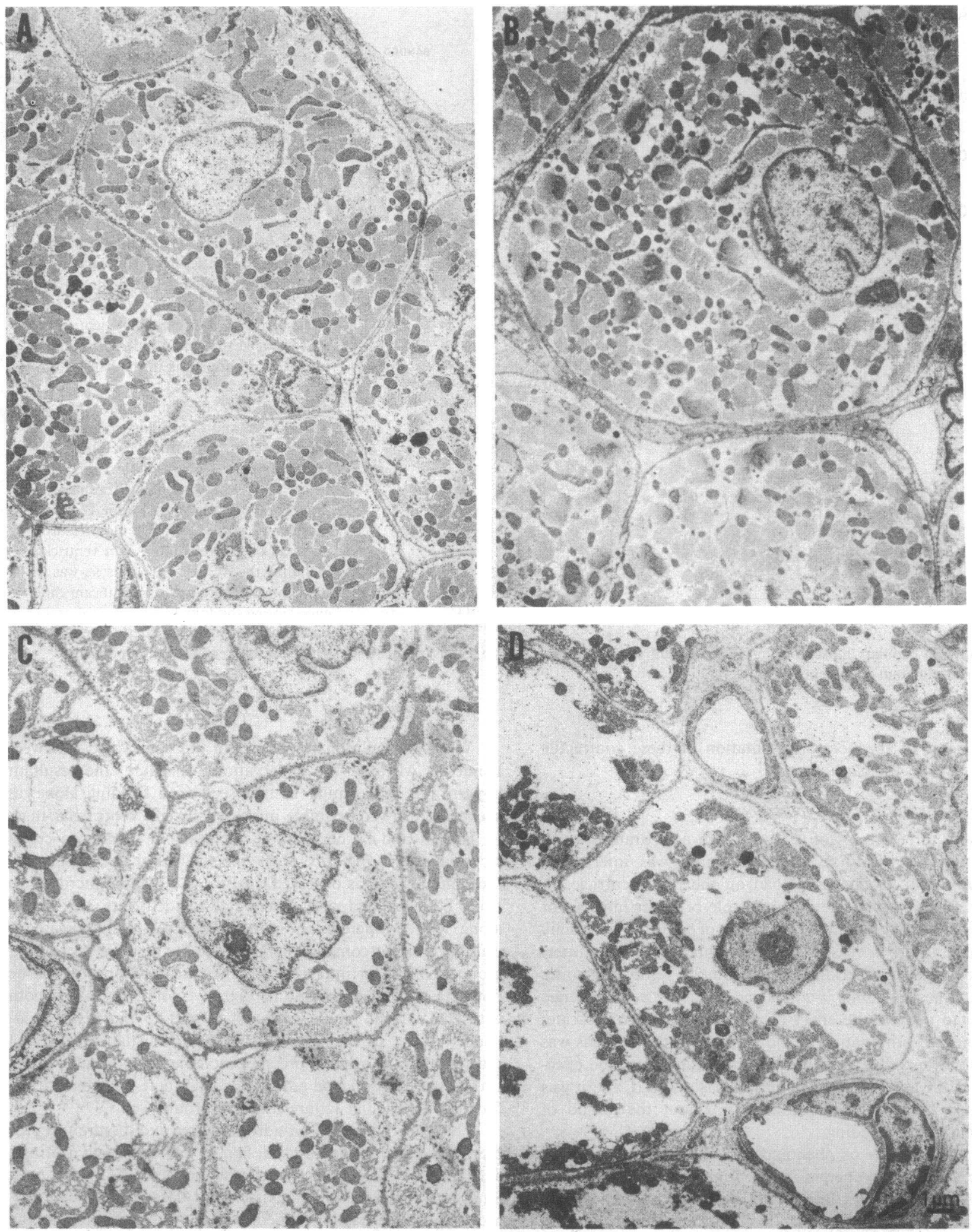

Figure 2. Electron micrographs of cross-sections from sham-operated control $(A)$, banded $(B)$, unloaded $(C)$, and unloaded-banded $(D)$ right ventricular papillary muscles obtained 2 wk after each surgical procedure. The hypertrophied cardiocytes of $B$ show an increase in

myofilaments, while the cardiocytes of $C$ and $D$ show an extensive loss of myofilaments resulting in large areas of organelle-free cytoplasm, with the remaining myofilaments present predominantly near the periphery of the cell. 
A

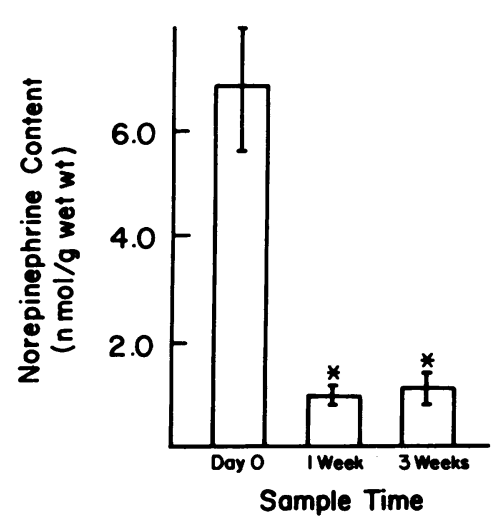

B

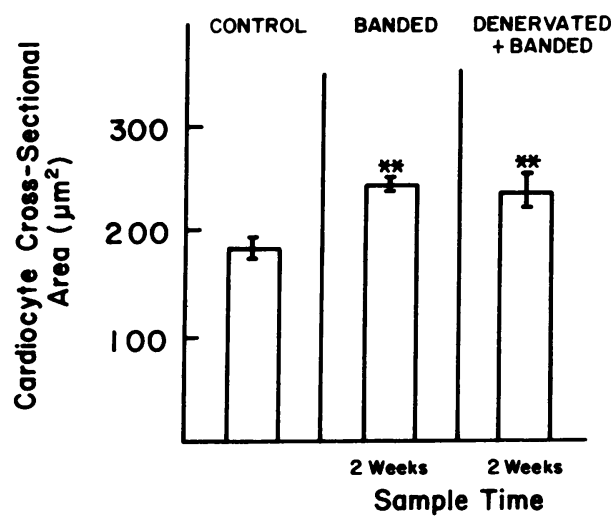

Figure 3. Evidence for right ventricular denervation and for right ventricular cardiocyte hypertrophy in cats in which the heart was denervated before pulmonary artery banding. $(A)$ Norepinephrine content in sequential right ventricular free wall biopsies obtained at the stated times from five cats. (B) Right ventricular cardiocyte size in papillary muscles from 10 control, seven banded, and five denervated-banded right ventricles. Each single asterisk in $A$ indicates a significant difference from the day zero value; there was no difference between the 1- and 3-wk samples. Each double asterisk in $B$ indicates a significant difference from the sham-operated controls. blockade then was compared with that of nonblocked, pulmonary artery-banded cats. As shown at the gross anatomical level in Table $I$ and at the cellular level in Fig. $5 \mathrm{~B}$, the degree of hypertrophy in each adrenoceptor blockade group was comparable with that in the group with banding alone. Again, there was no evidence, based on the data in Table I, for congestive heart failure in either adrenoceptor blockade group.

\section{Discussion}

This study, using adult mammalian cardiac preparations that respond rapidly and reversibly both to increased $(25,26)$ and to decreased $(4,5)$ loads, supports the hypothesis that increased load itself is the primary cause of cardiac hypertrophy in response to a hemodynamic overload. Two major lines of evidence support this statement: first, differential loading of two segments of the same ventricle, in the absence of any selective adrenergic intervention, results in changes in local tissue mass that are directionally the same as the changes in local tissue loading; second, when the influence of intrinsic or extrinsic catecholamines is excluded, the myocardial load responsiveness does not change.

\section{Differential loading}

The thesis that increased load might be a primary cause of cardiac hypertrophy has been addressed in terms of basic cardiac homeostasis. In a series of reviews of the factors thought to be responsible for the initiation of cardiac hypertrophy (27-29), a common theme was that each of these putative factors must necessarily act by altering the normal balance of cardiac protein synthesis and degradation in the direction of net protein synthesis. A more recent review (30) suggests that this balance is shifted primarily by an increase in protein synthesis, while the rate of protein degradation is largely unaffected.

Given this central role of augmented protein synthesis in the initiation of cardiac hypertrophy, it is of interest that an increased load on isolated heart muscle, in which variables other than loading conditions can be largely excluded, has been found to result in a very prompt increase in cardiac nuclear RNA polymerase activity (31) as well as in the protein synthesis of either contracting or quiescent preparations (1). In a recent study of protein synthesis in isolated hearts, it was found that increased cardiac load had a stimulatory effect on cardiac protein synthesis that could be dissociated from other potential contributors to this process (32). Finally, we have documented very rapid and substantial alterations in cardiac structure and function, which appear to be related almost entirely to changes in cardiac load in vivo $(4,5,25,26)$, and we have attributed these structural and functional changes primarily to changes in cardiac protein biochemistry, with reduced and then augmented cardiac loads being correlated with reduced and increased amounts of contractile proteins (33).

In this context, the reduction in cardiac tissue and cellular mass in response to a decrease in cardiac load, as documented in Table $\mathrm{I}$ and in Figs. $1 A$ and $2 C$, is a rather predictable experimental result. Similarly, the increased cardiac tissue and cellular mass in response to a pressure overload, as documented in Table $I$ and in Figs. $1 B$ and $2 B$, is also an expected experimental result. Of greater interest is the comparison of these data with the further data derived from myocardium unloaded in an otherwise overloaded and hypertrophying ventricle. With respect to the initiation of cardiac hypertrophy, the data in Figs. $1 C$ and $2 D$ demonstrate that when differential loading is imposed on two segments of the same ventricle in vivo, these two segments respond to local changes in load such that the unloaded segment atrophies in a hypertrophying ventricle. With respect to the maintenance of cardiac hypertrophy, the data in Fig. $1 D$ make the same point: local segments of the same ventricle respond to local loads with directionally appropriate changes in cardiocyte size. In addition to these structural changes, we examined in vitro the contractile function of both unloaded and overloaded papillary muscles from the same right ventricles in two cats in the unloadedbanded group using techniques which are standard in this laboratory (34). At the optimum length for tension generation, $L_{\max }$, the developed tension for normally loaded papillary muscles is $63.0 \pm 6.3 \mathrm{mN} / \mathrm{mm}^{2}$; for the unloaded papillary muscles, developed tension averaged $29.4 \mathrm{mN} / \mathrm{mm}^{2}$, a value very similar to the value of $25.4 \pm 5.7 \mathrm{mN} / \mathrm{mm}^{2}$ found in a study of papillary muscles unloaded in otherwise normally loaded cat right ventricles (4); the developed tension for the overloaded papillary muscles averaged $40.8 \mathrm{mN} / \mathrm{mm}^{2}$, a value very similar to the value of $41.5 \pm 2.7 \mathrm{mN} / \mathrm{mm}^{2}$ found in a study of papillary muscles from pressure-overloaded cat right 

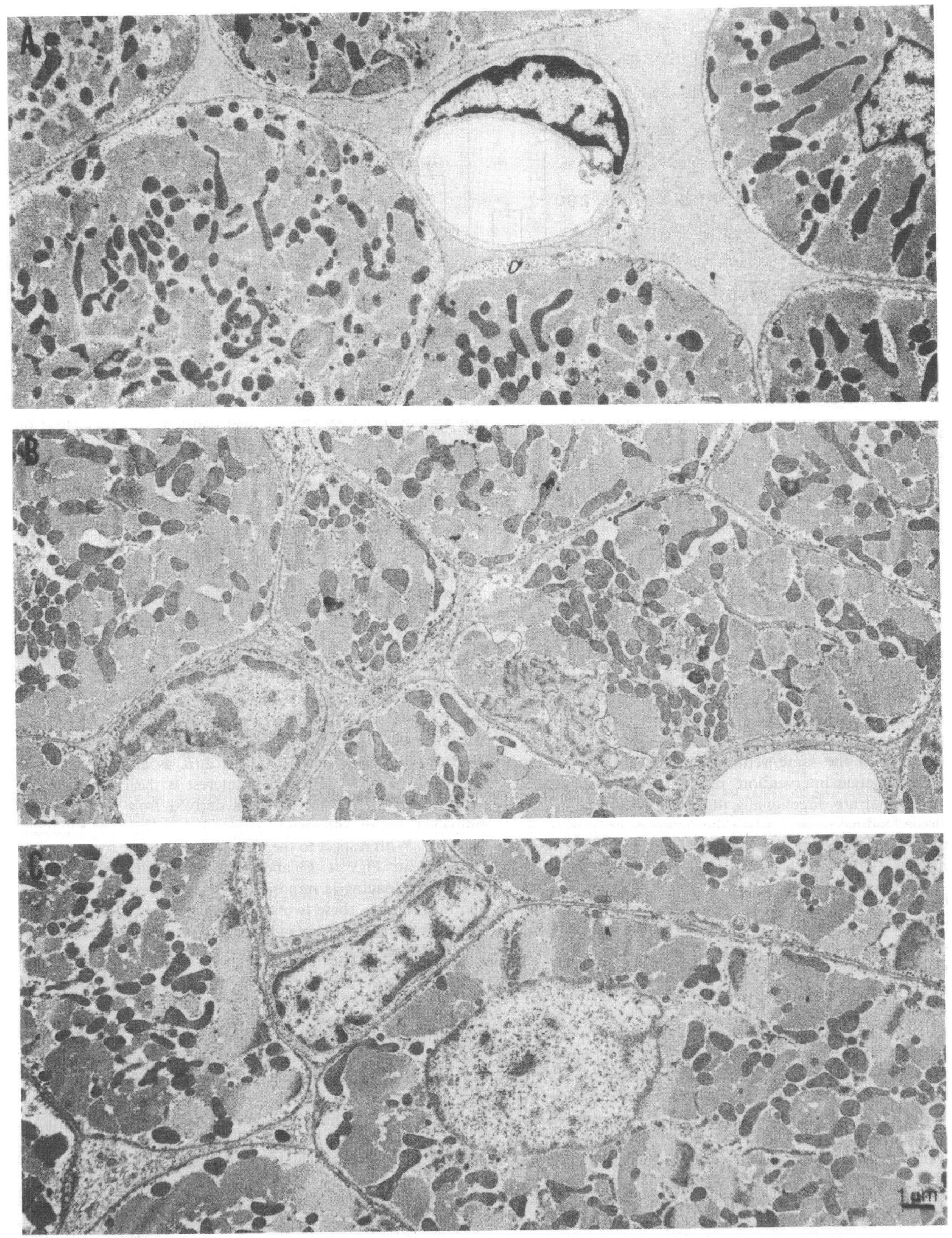

Figure 4. Electron micrographs of cross-sections from banded $(A)$, sham-operated control $(B)$, and denervated-banded $(C)$ right ventricular papillary muscles obtained 2 wk after each surgical procedure.
The cardiocytes in the upper and lower panels demonstrate a similar increase in size, and there is an apparent increase in connective tissue in the myocardium in $A$. 


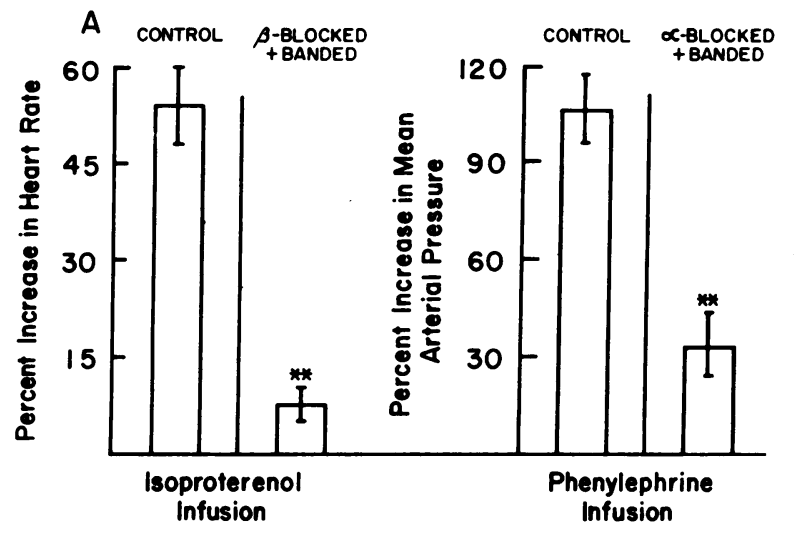

Figure 5. Evidence for cardiac $\beta$-adrenoceptor and $\alpha$-adrenoceptor blockade and for right ventricular cellular hypertrophy in cats in which each type of adrenoceptor blockade was begun before pulmonary artery banding. $(A)$ Response to a maximum pharmacological $\beta$ adrenoceptor or $\alpha$-adrenoceptor agonist challenge, in five cats in each instance, in which the corresponding prior adrenoceptor blockade had been induced before pulmonary artery banding. $(B)$ Right ventricular cardiocyte size in papillary muscles from ten sham-operated

ventricles (14). Thus, both the structural and functional characteristics of differentially loaded segments of the same right ventricle mimic those seen when underloading or overloading are imposed singly. Since we have not been able to postulate factors other than the immediate environment of cardiac stress and/or strain which might be responsible for these changes, we would suggest that these data constitute strong evidence for the primacy of increased load in the initiation and maintenance of cardiac hypertrophy in response to hemodynamic overloads.

\section{Adrenergic interventions}

The thesis that cardiac adrenoceptors, activated either by intrinsic cardiac nerves or by extrinsic circulating catecholamines, might be the common site through which a variety of factors initiating cardiac hypertrophy might act, has been and continues to be studied extensively. In the first instance, it is proposed that activation of the cardiac $\beta$-adrenoceptors via sympathetic nerve endings is the cause of cardiac hypertrophy; in the second instance, it is suggested that activation of these receptors by circulating adrenal catecholamines might be of greater importance. With respect to either adrenoceptor activation pathway, there are three major lines of investigation, apart from the present study, which may be used to evaluate the importance of the cardiac adrenoceptors in the initiation and maintenance of myocardial hypertrophy.

In the first line of investigation, neonatal rat cardiocytes in primary culture were used to evaluate cardiac hypertrophy. It was found in one of these studies (11) that both serum and norepinephrine cause cardiocyte hypertrophy. In the other study (12), it was concluded that the hypertrophic cardiocyte stimulation by norepinephrine was related to the $\alpha_{1}$ activity of this agent. However, there are two basic problems inherent in applying these results to the study of cardiac hypertrophy in the adult. First, as noted in a recent review (9), fetal heart cells differ fundamentally from adult heart cells with respect to growth regulation, in that fetal cardiocytes proliferate freely via hyperplasia, whereas adult cardiocytes can increase their mass solely through the mechanism of cellular hypertrophy. It

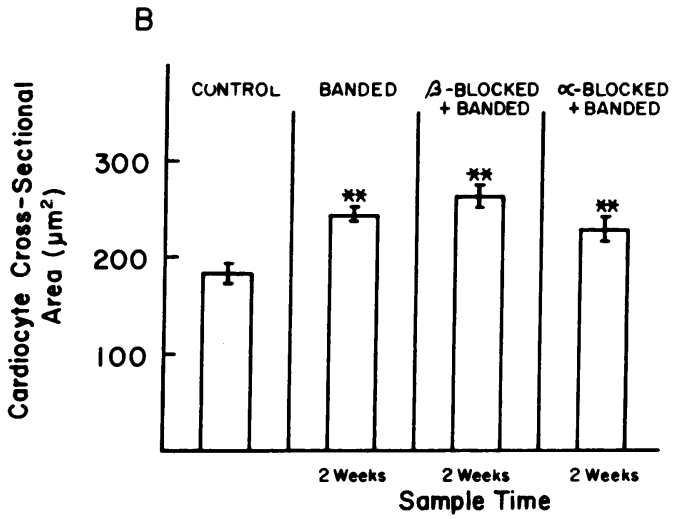

control and seven banded cats as well as in papillary muscles from five cats with $\beta$-adrenoceptor blockade and five cats with $\alpha$-adrenoceptor blockade before and during pulmonary artery banding. Each double asterisk in both $A$ and $B$ indicates a significant difference from the sham-operated control value. In addition, there was no difference in cardiocyte size among the banded, $\beta$-blocked, and $\alpha$ blocked groups.

would be surprising indeed if cells from a juvenile, growing, and developing heart were not responding to different stimuli from those acting on the adult heart, and there is direct evidence apart from the present study that this is the case: $\alpha$-adrenoceptor stimulation in adult rats prevents the cardiac hypertrophy ordinarily produced by a $\beta$-adrenoceptor agonist (35). Second, these fetal cells were contracting spontaneously, so that chronotropic and inotropic catecholamine effects on cell growth via increased cardiocyte stress and/or strain could not be separated from any direct catecholamine influence on cell growth.

The second line of investigation suggesting a role for catecholamines in cardiac hypertrophy has used primarily the spontaneously hypertensive rat model. In this genetic variant it has been suggested that adrenergic factors play an important role in modulating cardiac hypertrophy in response to increased systemic arterial pressure (36). However, several other lines of evidence suggest that this may not be the case. When 6hydroxydopamine was used to denervate the heart in another rat strain before inducing renal hypertension, the development of cardiac hypertrophy in response to this hemodynamic challenge was not prevented (37). Furthermore, both central and peripheral sympathectomy in the spontaneously hypertensive rat may serve to prevent the development of hypertension, but not the development of cardiac hypertrophy $(38-40)$. Thus, it would seem that the spontaneously hypertensive rat model has a genetic hypertrophic cardiomyopathy which is not strictly dependent on either catecholamines or systemic hypertension, while in a genetically distinct rat strain without spontaneous cardiac hypertrophy, cardiac enlargement can be induced by systemic hypertension, even in the absence of cardiac sympathetic nerves.

The third line of investigation in support of the role of catecholamines in cardiac hypertrophy is derived from direct infusions of $\beta$-adrenoceptor agonists into the circulation. For instance, it has been suggested (41) that an infusion of norepinephrine which did not cause systemic hypertension is sufficient to produce cardiac hypertrophy in the dog. However, in the absence of continuous concurrent measurements of heart 
rate and systemic arterial pressure during the time that hypertrophy was occurring, the contention that the increase in cardiac mass is unrelated to an increase in some net measurement of active ventricular wall stress, such as the tension-time index, is difficult to support. Indeed, in a study in the dog (10), it was found that the intrinsic catecholamine released in response to a pressure overload was predominantly the adrenal catecholamine epinephrine rather than norepinephrine, and it has been found in the rat that the catecholamines produced by the adrenal medulla are not necessary for the development of cardiac hypertrophy (42). Studies of chronic hypoxia, in which the hemodynamic load unfortunately was not defined, showed that right ventricular hypertrophy, at least in terms of organ weight, occurs despite either $\beta$-adrenoceptor blockade (2) or chemical sympathectomy (3). Finally, a study in dogs (43) showed that denervated hearts actually exhibit an accelerated hypertrophic response to pressure overloads.

The data in the present study demonstrate that catecholamines, whether intrinsic or extrinsic to the heart, are not necessary for the induction of cardiac hypertrophy in response to a pressure overload. In addition, given the quantitative similarity of the cardiac hypertrophic response to a pressure overload either with or without specific adrenergic interventions, the present data suggest that the adrenergic system does not substantially modify the cardiac hypertrophic response to a pressure overload. That is, when the intrinsic cardiac nerves are ablated, as documented in Fig. $3 A$, the hypertrophic response to a pressure overload shown in Figs. $3 B$ and $4 C$ does not differ from that same response in a normal heart. Similarly, when the response of the heart to extrinsic catecholamines is prevented by either $\beta$-adrenoceptor blockade or $\alpha$-adrenoceptor blockade, as documented in Fig. $5 \mathrm{~A}$, the tissue and cardiocyte response to a pressure overload, as shown in Table I and Fig. $5 B$, respectively, does not differ substantially from that observed in the absence of such blockade.

While it is clear from these data that catecholamines are not necessary for cardiac hypertrophy, the incidental denervation present in all of the surgical groups in this study, including the sham-operated cats, must raise the concern that if the cardiac innervation had been intact, the hypertrophic response might have been greater. An earlier study is germane to this point: we employed the same degree of pulmonary artery banding in the cat as that used here, but for $60 \mathrm{wk}$ before study (25), an interval greater than that reported to be required for the reinnervation of the hearts of cats (44) or dogs (45) after deliberate surgical cardiac denervation. The heart rate of these cats (25) during anesthesia with $25 \mathrm{mg} / \mathrm{kg}$ of ketamine, a phencyclidine derivative with centrally mediated sympathomimetic activity (46), was $181 \pm 7$ beats/min; for normal control cats in our laboratory after the same anesthesia a similar value of $186 \pm 9$ beats/min was obtained, but in the identically anesthetized cats with epicardial denervation in the present study, this value was significantly lower at $152 \pm 5$ beats/min, a value not greatly different from that of $\sim 110$ 140 beats/min which we see in awake, unrestrained cats. However, in the cats with prolonged pulmonary artery banding (25) and the above evidence of cardiac reinnervation, the degree of right ventricular hypertrophy was no greater than that observed either in the denervated group or in the other groups in this study.

\section{Conclusion}

Normal cardiac development, as well as growth throughout life, occur both in lower vertebrates such as the fish, whose hearts contain only $\sim 5 \%$ of the norepinephrine found in higher vertebrates, as well as in invertebrates such as the clam, in which only a negligibly small amount of cardiac catecholamines is found (24). Thus, the present finding in a higher vertebrate that catecholamines do not play a major primary role in cardiac hypertrophy in response to a pressure overload is not terribly surprising.

While the present data do not exclude a potentially important role for catecholamines in augmenting through inotropic and chronotropic effects the cardiac hypertrophic response to a pressure overload in vivo, the potential identification of cardiac adrenoceptor stimulation as a contributor to hypertrophy, independent of these effects, is rendered difficult by a major experimental problem: intrinsic to cardiac catecholamine activity are increases in the extent of contraction, the velocity of contraction, and the frequency of contraction. Since each of these three factors varies directly with the amount of both active and passive cardiac stress and strain per unit of time, the identification of any primary catecholamine stimulation of hypertrophy separate from a secondary contribution to hypertrophy via an increase in one or more elements of cardiac load would seem to be virtually impossible in a contracting preparation.

With respect to other potential trophic factors, it has been suggested recently that there may be an as yet undefined watersoluble molecule that induces hypertrophy in unloaded myocardium (13). In addition, while some minimum level of adrenocorticoids clearly plays a permissive role in cardiac hypertrophy (42), as might be expected from long-standing observations of reduced cardiac mass in Addison's disease, and the cardiac cytosolic receptor number for glucocorticoids may be increased in some forms of cardiac hypertrophy (47), other data suggest that a primary hypertrophy-inducing steroid is secreted by the adrenal cortex (8). The data in the present study derived from differential loading conditions would suggest that any such circulating molecule, which would necessarily be present in all myocardial regions, must somehow act selectively in the present model only on myocardial regions in which the hemodynamic load is increased. Also, data demonstrating load-stimulated cardiac protein synthesis by superfused preparations in vitro (1) would dictate that any such substance be elaborated by the same cardiocytes on which it is to act. Finally, it would be necessary to show for any such trophic substance either that it acts on quiescent cardiac preparations or that it does not increase cardiac load through inotropic and chronotropic effects.

Apart from the identification of load as a primary regulator of cardiac structure and function, provided both by this study and by our earlier studies $(4,5,25,26)$, the means by which an increased hemodynamic load serves as the primary stimulus to cardiac hypertrophy is of considerable further basic interest. Two intriguing mechanistic possibilities derive from the fact that an early response to hemodynamic overloading is an increase in diastolic cardiac segment length. This length change could provide a signal for greater cardiocyte protein synthesis via either the increased aerobic metabolism (34) or the increased cyclic nucleotide synthesis (48) which a length increase entails, 
or through some as yet unidentified pathway. The elucidation of the one or several mechanisms by which load is transduced into growth in adult mammalian myocardium remains as one of the most important problems to be addressed in normal and abnormal cardiac physiology.

\section{Acknowledgments}

The authors wish to thank Thomas Vinciguerra, Stephen Vinciguerra, and Joseph Severdia for their technical assistance with these studies. We also wish to thank Jane Axamethy for preparing the graphics.

This work was supported by grants HL 07198, HL 29146, HL 29351, and HL 29718 from the National Institutes of Health, by grant 82-800 from the American Heart Association, and by the Pennsylvania and Delaware Heart Associations.

\section{References}

1. Peterson, M. B., and M. Lesch. 1972. Protein synthesis and amino acid transport in the isolated rabbit right ventricular papillary muscle. Circ. Res. 31:317-327.

2. Dennis, P., and E. M. Vaughan Williams. 1982. Hypoxic cardiac hypertrophy is not inhibited by cardioselective or non-selective $\beta$ adrenoceptor antagonists. J. Physiol. 324:365-374.

3. Vaughan Williams, E. M., and I. D. Dukes. 1983. The absence of effect of chemical sympathectomy on ventricular hypertrophy induced by hypoxia in young rabbits. Cardiovasc. Res. 17:379-389.

4. Cooper, G., and R. J. Tomanek. 1982. Load regulation of the structure, composition and function of mammalian myocardium. Circ. Res. 50:788-798.

5. Thompson, E. W., T. A. Marino, C. E. Uboh, R. L. Kent, and G. Cooper. 1984. Atrophy reversal and cardiocyte redifferentiation in reloaded cat myocardium. Circ. Res. 54:367-377.

6. Ostman-Smith, I. 1979. Adaptive changes in the sympathetic nervous system and some effector organs of the rat following long term exercise or cold acclimation and the role of cardiac sympathetic nerves in the genesis of compensatory cardiac hypertrophy. Acta Physiol. Scand. 108(Suppl. 477):1-118.

7. Ostman-Smith, I. 1981. Cardiac sympathetic nerves as the final common pathway in the induction of adaptive cardiac hypertrophy. Clin. Sci. 61:265-272.

8. Kolbel, F., and V. Schreiber. 1983. Biochemical regulators in cardiac hypertrophy. Basic Res. Cardiol. 78:351-363.

9. Zak, R. 1984. Factors controlling cardiac growth. In Growth of the Heart in Health and Disease. R. Zak, editor. Raven Press, New York. 165-185.

10. Womble, J. R., M. K. Haddox, and D. H. Russell. 1978. Epinephrine elevation in plasma parallels canine cardiac hypertrophy. Life Sci. 23:1951-1958.

11. Simpson, P., A. McGrath, and S. Savion. 1982. Myocyte hypertrophy in neonatal rat heart cultures and its regulation by serum and by catecholamines. Circ. Res. 51:787-801.

12. Simpson, P. 1983. Norepinephrine-stimulated hypertrophy of cultured rat myocardial cells is an $\alpha_{1}$ adrenergic response. J. Clin. Invest. 72:732-738.

13. Hammond, G. L., Y. K. Lai, and C. L. Markert. 1982. The molecules that initiate cardiac hypertrophy are not species-specific. Science (Wash. DC). 216:529-531.

14. Cooper, G., R. M. Satava, C. E. Harrison, and H. N. Coleman. 1973. Mechanism for the abnormal energetics of pressure-induced hypertrophy of cat myocardium. Circ. Res. 33:213-223.

15. Kaye, M. P., G. G. Brynjolfsson, and W. P. Geis. 1968 Chemical epicardiectomy. Cardiologia. 53:139-149.

16. Martins, J. B., and D. P. Zipes. 1980. Epicardial phenol interrupts refractory period responses to sympathetic but not vagal stimulation in canine left ventricular epicardium and endocardium. Circ. Res. 47:33-40.

17. Marino, T. A., S. R. Houser, and G. Cooper. 1983. Early morphological alterations of pressure overloaded cat right ventricular myocardium. Anat. Rec. 207:417-426.

18. Marino, T. A., S. R. Houser, F. G. Martin, and A. R. Freeman. 1983. An ultrastructural morphometric study of the papillary muscle of the right ventricle of the cat. Cell Tissue Res. 230:543-552.

19. Watson, M. L. 1958. Staining of tissue sections for electron microscopy with heavy metals. J. Biophys. Biochem. Cytol. 4:475-478.

20. Reynolds, E. S. 1963. The use of lead citrate at high $\mathrm{pH}$ as an electron opaque stain in electron microscopy. J. Cell. Biol. 17:208213.

21. Keller, R., A. Oke, I. Mefford, and R. N. Adams. 1976. Liquid chromatographic analysis of catecholamines. Life Sci. 19:995-1004.

22. Felice, L. J., J. D. Felice, and P. T. Kissinger. 1978. Determination of catecholamines in rat brain parts by reverse-phase ion-pair liquid chromatography. J. Neurochem. 31:1461-1465.

23. Anton, A. H., and D. F. Sayre. 1962. A study of the factor affecting the aluminum oxide-trihydroxyindole procedure for the analysis of catecholamines. J. Pharmacol. Exp. Ther. 138:360-375.

24. Lee, W. C., J. M. Lew, and C. S. Yoo. 1970. Studies on myocardial catecholamines related to species ages and sex. Arch. Int. Pharmacodyn. 185:259-268.

25. Cooper, G., R. J. Tomanek, J. C. Ehrhardt, and M. L. Marcus. 1981. Chronic progressive pressure overload of the cat right ventricle. Circ. Res. 48:488-497.

26. Cooper, G., and T. A. Marino. 1984. Complete reversibility of cat right ventricular chronic progressive pressure overload. Circ. Res. 54:323-331.

27. Rabinowitz, M. 1974. Overview on pathogenesis of cardiac hypertrophy. Circ. Res. 34/35(Suppl. II):3-11.

28. Zak, R. 1974. Development and proliferative capacity of cardiac muscle cells. Circ. Res. 34/35(Suppl. II):17-26.

29. Morkin, E. 1974. Activation of synthetic processes in cardiac hypertrophy. Circ. Res. 34/35(Suppl. II):37-48.

30. Schreiber, S. S., C. D. Evans, M. Oratz, and M. A. Rothschild. 1981. Protein synthesis and degradation in cardiac stress. Circ. Res. 48:601-611.

31. Schreiber, S. S., M. Oratz, and M. A. Rothschild. 1969. Nuclear RNA polymerase activity in acute hemodynamic overload in the perfused heart. Am. J. Physiol. 217:1305-1309.

32. Kira, Y., P. J. Kochel, E. E. Gordon, and H. E. Morgan. 1984. Aortic perfusion pressure as a determinant of cardiac protein synthesis. Am. J. Physiol. 246:C247-C258.

33. Kent, R. L., C. E. Uboh, E. W. Thompson, S. S. Gordon, T. A. Marino, J. K. Hoober, and G. Cooper. 1984. Biochemical and structural correlates in unloaded and reloaded cat myocardium. $J$. Mol. Cell. Cardiol. In press.

34. Cooper, G. 1981. Influence of length changes on myocardial metabolism in the cat papillary muscle. Circ. Res. 49:423-433.

35. Pagano, V. T., and M. A. Inchiosa. 1977. Cardiomegaly produced by chronic $\beta$-adrenergic stimulation in the rat: comparison with $\alpha$-adrenergic effects. Life Sci. 21:619-624.

36. Sen, S., and R. C. Tarazi. 1983. Regression of myocardial hypertrophy and influence of adrenergic system. Am. J. Physiol. 244: H97-H101.

37. Cohen, J. 1974. Role of endocrine factors in the pathogenesis of cardiac hypertrophy. Circ. Res. 34/35(Suppl. II):49-57.

38. Cutilletta, A. F., L. Erinoff, A. Heller, J. Low, and S. Oparil. 1977. Development of left ventricular hypertrophy in young spontaneously hypertensive rats after peripheral sympathectomy. Circ. Res. 40:424-434.

39. Oparil, S., and A. F. Cutilletta. 1979. Hypertrophy in the denervated heart: a comparison of central sympatholytic treatment 
with 6-hydroxydopamine and peripheral sympathectomy with nerve growth factor antiserum. Am. J. Cardiol. 44:970-978.

40. Tomanek, R. J., R. K. Bhatnagar, P. Schmid, and M. J. Brody. 1982. Role of catecholamines in myocardial cell hypertrophy in hypertensive rats. Am. J. Physiol. 242:H1015-H1021.

41. Laks, M. M., F. Morady, and H. J. C. Swan. 1973. Myocardial hypertrophy produced by chronic infusion of subhypertensive doses of norepinephrine in the dog. Chest. 64:75-78.

42. Nichols, J. R., R. L. Clancy, and N. C. Gonzalez. 1983. Role of adrenals on development of pressure-induced myocardial hypertrophy. Am. J. Physiol. 244:H234-H238.

43. Matoba, T., K. Adachi, T. Ito, Y. Yamashita, M. Chiba, K. Odawara, S. Inuzuka, and H. Toshima. 1984. Cardiac hypertrophy in surgically denervated dogs with aortic stenosis. Experientia (Basel). 40: 73-75.

44. Jacobowitz, D., T. Cooper, and H. B. Barner. 1967. Histochem- ical and chemical studies of the localization of adrenergic and cholinergic nerves in normal and denervated cat hearts. Circ. Res. 20:289-298.

45. Kaye, M. P., W. C. Randall, G. R. Hageman, W. P. Geis, and D. V. Priola. 1977. Chronology and mode of reinnervation of the surgically denervated canine heart: functional and chemical correlates. Am. J. Physiol. 233:H431-H437.

46. White, P. F., W. L. Way, and A. J. Trevor. 1982. Ketamineits pharmacology and therapeutic uses. Anesthesiology. 56:119-136.

47. Hickson, R. C., T. M. Galassi, T. T. Kurowski, D. G. Daniels, and R. T. Chatterton. 1984. Androgen and glucocorticoid mechanisms in exercise-induced cardiac hypertrophy. Am. J. Physiol. 246:H761H767.

48. Singh, J. 1982. Stretch stimulates cyclic nucleotide metabolism in the isolated frog ventricle. Pfluegers Arch. Eur. J. Physiol. 395:162164. 